\title{
Desired Dental Treatments for Discoloured Anterior Teeth at Medunsa Oral Health Centre, South Africa
}

\author{
Pumela Gwengu* \\ Sefako Makgatho Health Science University, South Africa
}

Submission: September 14, 2017; Published: April 30, 2018

*Corresponding author: Pumela Gwengu, Sefako Makgatho Health Science University, South Africa, Tel: 5214827; Email: pumela26@gmail.com

\begin{abstract}
Summary
Background: People are generally concerned about their dental appearance and, therefore demand cosmetic dental services. Patients demand not only a healthy mouth but also what they consider a perfect smile. A healthy, bright and beautiful smile does have an impact on individual beauty, self-consciousness, self-image as well as self-confidence. Desired dental treatment for discoloured anterior teeth is a reflection of individual preferences, socio-demographic and cultural practices. It may also relate to the type of treatment that the patient may afford. Objectives: To investigate factors that influence patients` desired dental treatment need for their discoloured anterior teeth.
\end{abstract}

Methodology: A total of 70 patients who attended a dental training school at Medunsa Oral Health Centre in Pretoria, South Africa participated in the study. A researcher-administered questionnaire containing questions about socio-demographic variables and desired dental treatment needs was used to collect data. A descriptive cross-sectional study was conducted, and frequency counts, percentage calculations, together with $95 \%$ confidence levels were summarised. Percentage outcomes were compared by Fisher`s exact test. All statistical tests were twosided and $\mathrm{p}$ values 0.05 were considered significant.

Results: According to the research findings, $96 \%$ of the participants were aware of their discoloured anterior teeth, $77 \%$ embarrassed, $93 \%$ dissatisfied, and 69\% experienced negative comments from communities about their discoloured anterior teeth. Most participants (54\%) had secondary education, $31 \%$ had tertiary education, and $38 \%$ were unemployed. Some participants $(43 \%)$ reported that they experienced dental pain, $76 \%$ tooth sensitivity and $76 \%$ stated that they had trust in the dental professionals, and also had hope that their main complaints will be addressed and managed. Most participants (52\%) considered tooth whitening as the best option, and tooth extraction without replacement was not an option for most (50\%) of them. Placement of crowns was unpopular to some (20\%), as they! were unsure and knew nothing about them.

Conclusion: The majority of the participants were aware and dissatisfied with the colour of their anterior and most of them requested tooth whitening.

Keywords: Discoloured anterior teeth; Desired dental treatments

Abbreviations: MOHC: Medunsa Oral Health Centre; SMU: Sefako Makgatho Health Sciences University

\section{Introduction}

Tooth shape, size, appearance and colour are a concern to many people who seek dental treatment. Tooth discolouration, dental fluorosis in particular, or any other causative factors such as dental caries, tetracycline stains, certain other drugs and dental trauma cause significant cosmetic problems, especially when it affects the anterior teeth. Tooth discolouration is the staining of teeth that occurs when the enamel and dentine become discoloured. As a result, tooth discolouration is aesthetically displeasing and is a psychological challenge to patients since it detracts or erodes the sparkle from a smile [1].

The colour of ones teeth may vary from white to creamyyellow and it differs from one individual to another. Robinson [2] stated that the colour of teeth is an important feature in determining the attractiveness of the face [2]. Therefore, any form of unsightly tooth stains may cause great embarrassment to individuals and can also stop people from smiling. Altered or spoiled tooth colour does affect the appearance of the dentition and it is a great concern to a large number of individuals who seek dental care at oral health institutions [3]. As a result, perceptions of tooth appearance in a modern society can influence changes in patient's subjective needs [4].

Patients are continuously exposed to various sources of professional and non-professional health knowledge or health advice. This health advice may lead to development of selfawareness about oral conditions. Any health-related information, knowledge, and advice may come from friends, family, neighbours, the media, as well as health care professionals. It can be this wealth of knowledge or some form of illness, dental pain or discomfort that lead to the development of a patient's healthcare-seeking behaviour. Patients seek oral health services for both cosmetic and health reasons, and in so doing often express their health needs in relation to their oral problems. 


\section{Advances in Dentistry \& Oral Health}

Medunsa Oral Health Centre (MOHC) is a tertiary health-care facility that renders oral health services in a multidisciplinary approach for at least 8-9hrs a day, for 5 days in a week. On Saturdays emergency oral health services are provided i.e. for the relief of pain and sepsis.

Results

Table 1:

\begin{tabular}{|c|c|c|}
\hline Variables & $\mathbf{N}=70$ & Percentages \\
\hline Age groups $(<30$, Mean age $=28$, Range $14-53)$ & 48 & $69 \%$ \\
\hline Awareness of their tooth discolouration, Embarrassed & 67,54 & $96 \%, 77 \%$ \\
\hline Secondary education, Tertiary qualification & 38,22 & $54 \%, 31 \%$ \\
\hline Urban areas, Unemployed & 44,27 & $63 \%, 38 \%$ \\
\hline Dissatisfied, Experienced negative comments & 65,48 & $93 \%, 69 \%$ \\
\hline Previous dental visits & Yes (34), No (36) & $49 \%, 51 \%$ \\
\hline Dental pain, Tooth sensitivity & 30,53 & $43 \%, 76 \%$ \\
\hline Trust in dental professional & 53 & $76 \%$ \\
\hline
\end{tabular}

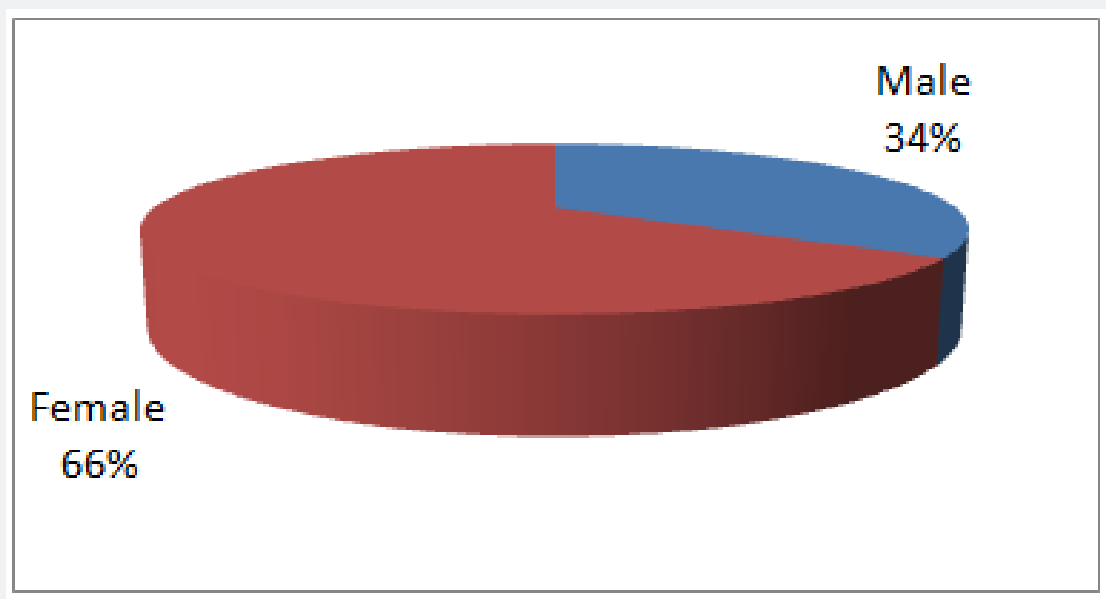

Figure 1: Distribution of gender.

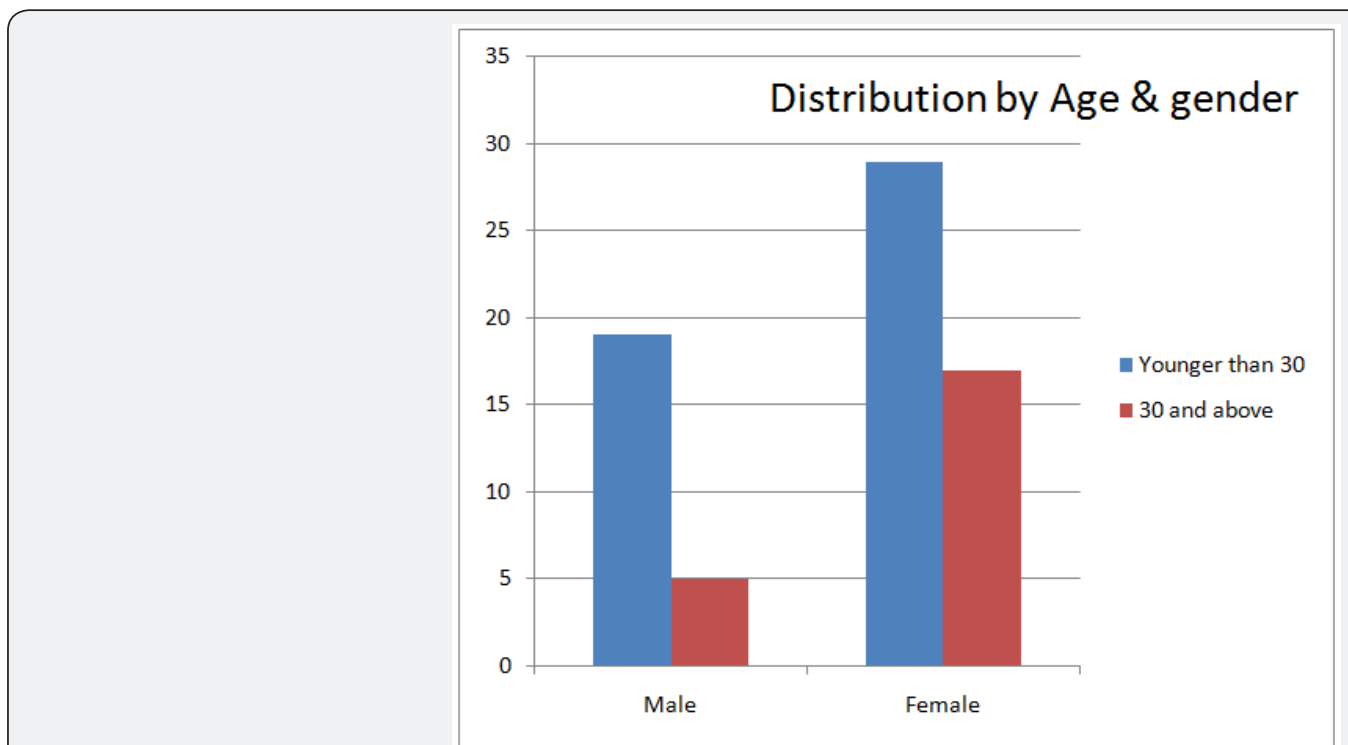

Figure 2: Distribution by age and gender. 
A total of 70 patients at a tertiary oral health training facility in Pretoria in South Africa participated in the study. There were 46 females and 24 males (Figure 1), with an age range of 14 - 53, mean age 28 (Table 1). According to the findings, 69\% were less than 30 years of age (Figure 2), 96\% were aware of their discoloured anterior teeth, 77\% embarrassed, 93\% dissatisfied and 69\% experienced negative comments from communities about the colour of their teeth. Most participants (54\%) had secondary education, 31\% tertiary education and 38\% were unemployed. Some participants (43\%) reported that they experienced dental pain, $76 \%$ tooth sensitivity, and $51 \%$ had no previous dental visits. Seventy-six percent stated that they had trust in the dental professionals, hoping that their main complaints would be addressed and managed.

Majority of the participants (77\%) reported that they were in need of tooth whitening, $71 \%$ preferred tooth coloured restorative fillings and $66 \%$ disliked false teeth. Most of them (54\%) were unsure of dental crowns. Likewise, $66 \%$ of the participants said no to tooth extraction without replacement and $54 \%$ were not sure of dental implants (Figure 3). Most participants (52\%) younger than 30 years were interested in tooth whitening compared to their counterparts in the older age group (24\%) (Figure 4). Some of them $(48 \%)$ were in need of tooth coloured restorative fillings compared to their counterpart (23\%). Tooth extraction without replacement of anterior teeth was not an option for most participants (50\%) compared to the $4 \%$ who agreed. A small group (47\%) refused false teeth as compared to the $6 \%$ of those who reported yes. Some participants (34\%) were unsure of dental crowns compared to their counter parts (20\%). Only 6\% of the older group (above 30 years) said yes to dental implants, $34 \%$ were not sure and $29 \%$ said no to dental implants. None of these percentage outcomes that were reported in this study were statistically significant (Figure 5).

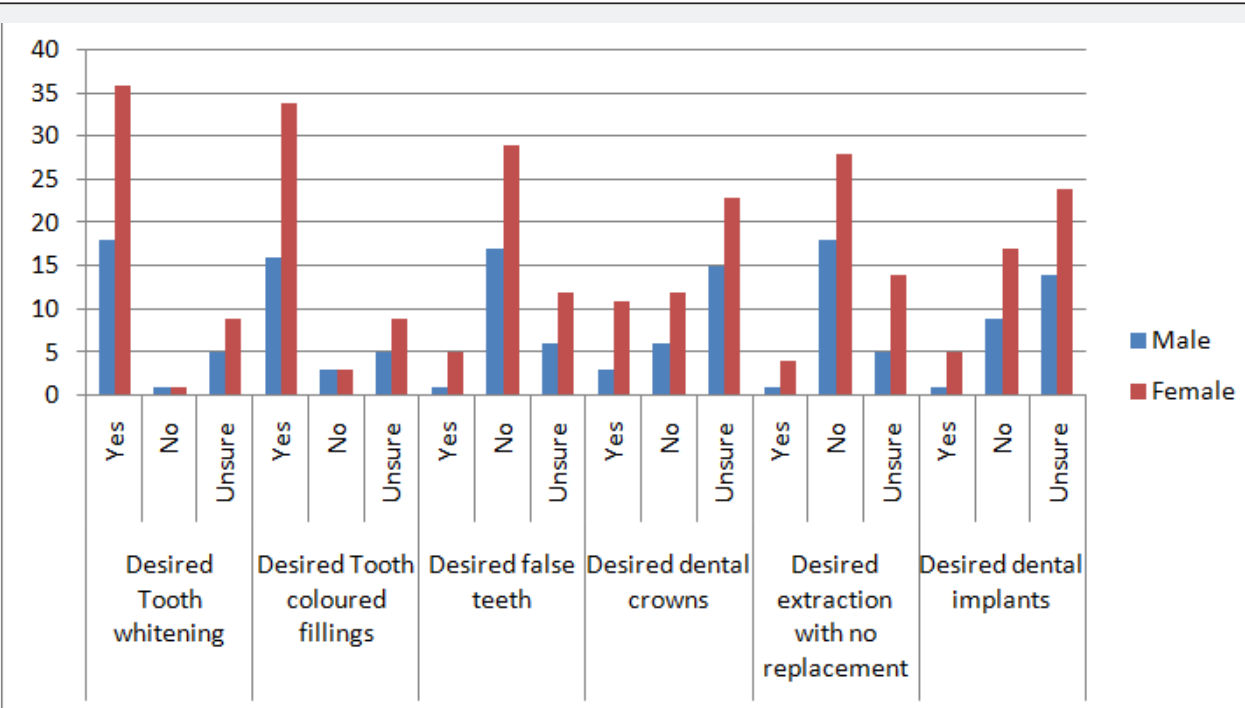

Figure 3: Distribution by gender and desired dental treatment.

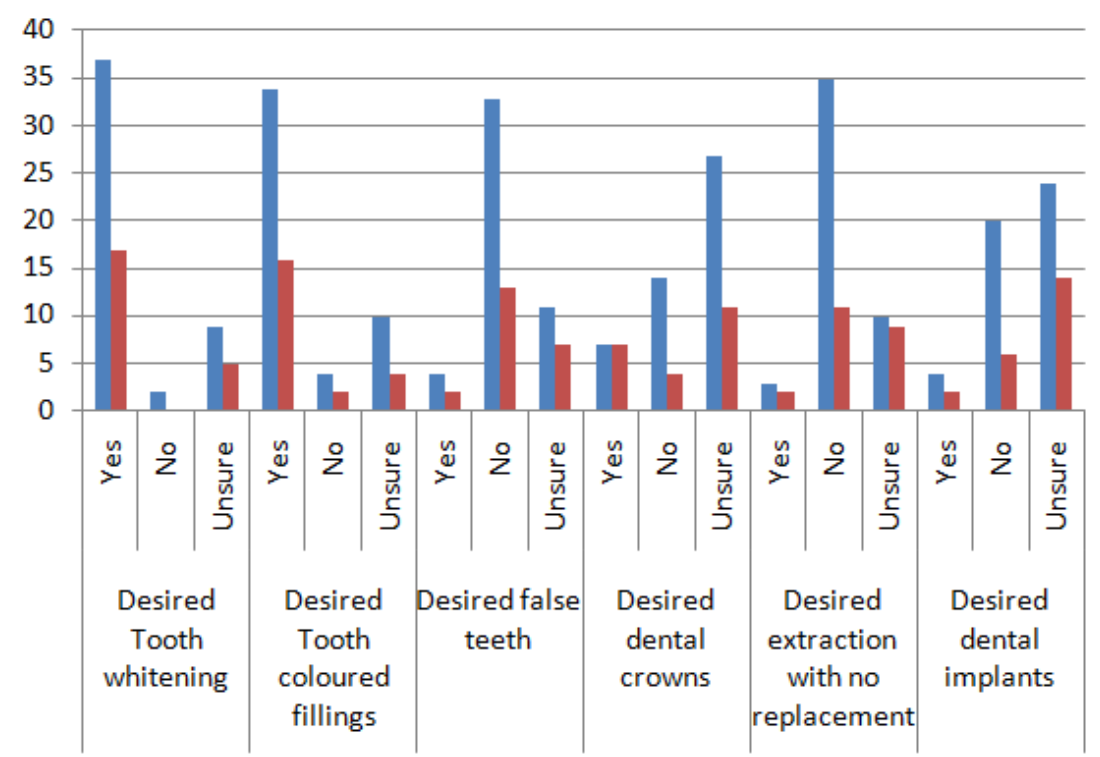

Figure 4: Distribution by age group and desired dental treatment. 


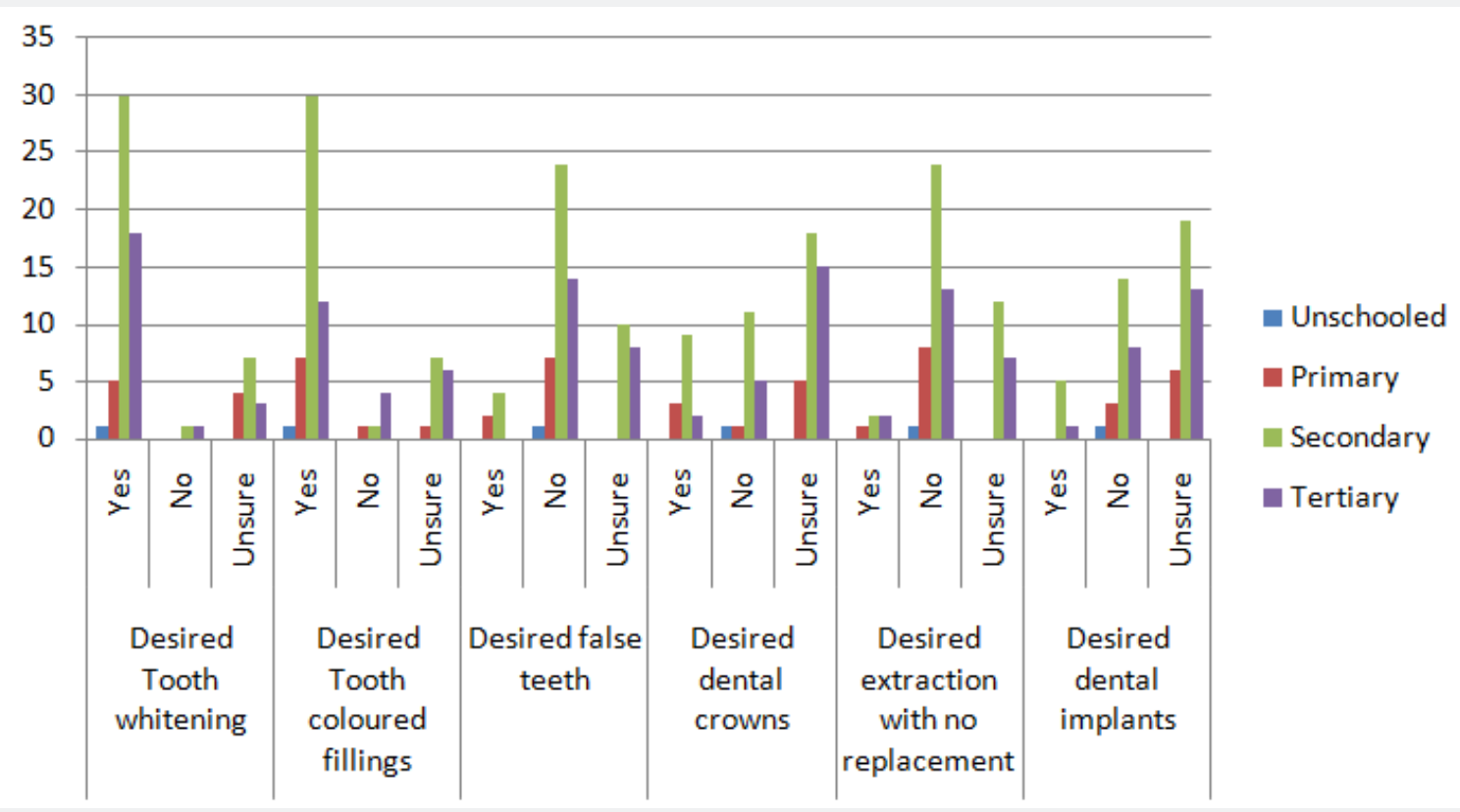

Figure 5: Distribution by level of education and desired dental treatment.

\section{Discussion}

Seventy patients participated in the study of which $66 \%$ were females and 34\% were male. Majority of the participants were aware, dissatisfied and embarrassed about their discoloured anterior teeth. A study by Tin-Oo [5] and other researchers concluded that most patients were not satisfied with their dental appearance and therefore demanded improvement in aesthetics [5]. They further noted that unhappiness with tooth colour was mostly reported by females [5]. Ibiyeni \& Taiwo [3] also identified that discolouration (non-aesthetic) of anterior teeth produces profound embarrassment and psychological distress to patients [3]. Previous study by Watts \& Addy [6] stated that anterior tooth discoloration is one of the most important and frequent reasons for seeking dental treatment [6]. Well and above, different authors [7] noted that there was a general dissatisfaction about tooth discolouration amongst members of the public, of which they recommended appropriate management of such patients [7].

Majority of the participants had tooth sensitivity, some had dental pain, others unemployed and some had no previous dental visits.

Majority of the participants (77\%) reported that they were in need of tooth whitening, mainly females who were younger than 30 years of age. The study findings are in agreement with that of Alkhatib [7] and others which indicated that people are generally concerned about their dental appearance and, therefore demand cosmetic dental services like tooth whitening [7]. A study by Akarslan [8] and others further noted that the higher dissatisfaction level for females could be related to the idea of their self-esteem, health awareness behaviour, or any other physical conditions that can affect them more than males [8]. Females were more dissatisfied with the general appearance of their teeth compared to males in this study.
There was no particular sequence in terms of management of discoloured anterior teeth but the majority of the participants (77\%) reported that they were in need of tooth whitening $71 \%$ preferred tooth coloured restorative fillings and $66 \%$ disliked false teeth. Most of them (54\%) were unsure of dental crowns, likewise $66 \%$ of them said no to tooth extraction without replacement and $54 \%$ were not sure of dental implants. A study carried by Samorodnitzky-Naveh [4] and others in 2007 also noted that most patients find the presence of six anterior teeth indispensable and can accept edentulous spaces only in the posterior region [4].

Contrary to previous study by Klages \& Zentner [9] who stated that some patients mentioned a particular order of what they would prefer in management of their discoloured anterior teeth such as;

1. Aesthetic restorations that match their smile and facial complexion;

2. Root canal treatments for badly decayed anterior teeth;

3. Prosthetic restorations;

4. Tooth whitening and lastly;

5. Dental extraction [9].

Another study by Samorodnitzky-Navehand [4] others also reported that the most-desired basic treatment for discoloured anterior teeth was found to be whitening of teeth $(49.0 \%)$, followed by aesthetic restorations (25.4\%), orthodontic treatment (24.5\%) and prosthetic restorations (16.9\%) [4].

Majority of the participants (54\%) had secondary education, $96 \%$ were aware of their discoloured anterior teeth, and 77\% were embarrassed. Educational levels had an impact on the general appearance of dental aesthetics in the present study, but participants with secondary education were more interested in 
tooth whitening and tooth coloured restorations as compared to those who were unschooled or with little education. A study by Akarslan [8] and others reflected that the appearance of the teeth could be influenced by gender, age and educational level [8]. They further reported that females were more sensitive than males about the appearance of teeth, and the importance of teeth decreases with ageing and higher education levels [8]. Their study emphasised gender, age and educational levels, which had an effect on received previous dental treatments on anterior teeth and desired treatments for improvement of aesthetic [8].

Previous research work by Akarslan [8] and others reflected that, with the increase in the education level, the dissatisfaction with tooth colour decreased [8]. Education is one way by which selfesteem may be enhanced. Thus, it is possible that the attainment of higher education may indirectly improve self-satisfaction with tooth colour [9]. Another study by Xiao [10] and others reported that dental aesthetic satisfaction in a Chinese population was correlated with education level, but not with age and gender [10].

Under normal circumstances all these treatment options depend on socio-demographic and socio-economic factors, the availability of time, distance to treatment centres and the balancing of family responsibilities. A major goal of managing patients in dentistry is to re-establish beauty, functionality and to enable patients to feel confident about their facial appearance.

In this study, the most desired dental treatment by most participants was tooth whitening. Understanding the desired treatments for discoloured anterior teeth can guide health professionals on strategies for intervention and how to improve dental aesthetics.

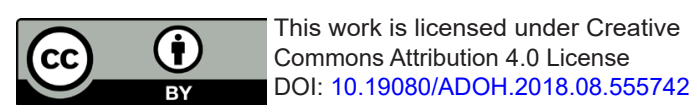

\section{Acknowledgements}

Authors are happy to thank all healthcare professionals at Sefako Makgatho Health Sciences University (SMU) for their sterling contributions to the piece of knowledge and science behind the research work.

\section{References}

1. Manuel ST, Abhishek P, Kundabala M (2010) Etiology of tooth discolouration- a review. Nigerian Dental Journal 18(2): 56-63.

2. Robinson PG (2008) The influence of tooth colour on perceptions of personal characteristics among female dental patients: comparisons of unmodified, decayed and whitened teeth. Br Dent J 204(5): 256-257.

3. Ibiyeni O, Taiwo JO (2011) Psychosocial aspect of anterior tooth discolouration among adolescents in Igbo-Ora, South Western Nigeria. Annals of Ibadan Postgraduate Medicine 9 (2): 94-99.

4. Samorodnitzky- Naveh GR, Geiger SB, Levin L (2007) Patient's satisfaction with dental aesthetics. JADA 138(6): 805-808.

5. Tin-Oo MM, Saddki N, Hassan, N (2011) Factors influencing patient satisfaction with dental appearance and treatment they desired to improve aesthetics. BMC Oral Health 11: 6.

6. Watts A, Addy M (2001) Tooth discolouration and staining: a review of the literature. Br Dent J 190(6): 309-316.

7. Alkhatib MN, Holt R, Bedi R (2004) Prevalence of self -assessed tooth discolouration in the United Kingdom. Journal of Dentistry 32(7): 561566.

8. Akarslan ZZ, Sadik B, Erten H, Karabulut E (2009) Dental aesthetic satisfaction received and desired dental treatments for improvement of aesthetics. Indian Journal of Dental Research 20(2): 195-200.

9. Klages U, Zentner A (2007) Dentofacial Aesthetics and Quality of life. Seminars in Orthodontics 13(2): 104-115.

10. Xiao J, Zhou XD, Zhu WC, Zhang B, Li JY, et al. (2007) The prevalence of tooth discolouration and the self-satisfaction with tooth colour in a Chinese urban population. J Oral Rehabil 34(5): 351-360.

\section{Your next submission with Juniper Publishers will reach you the below assets}

- Quality Editorial service

- Swift Peer Review

- Reprints availability

- E-prints Service

- Manuscript Podcast for convenient understanding

- Global attainment for your research

- Manuscript accessibility in different formats

( Pdf, E-pub, Full Text, Audio)

- Unceasing customer service

Track the below URL for one-step submission https://juniperpublishers.com/online-submission.php 